\title{
Interacting two helical edge modes in quantum spin Hall systems
}

\author{
Yukio Tanaka ${ }^{1}$, and Naoto Nagaosa ${ }^{2,3}$ \\ ${ }^{1}$ Department of Applied Physics, Nagoya University, Nagoya, 464-8603, Japan \\ 2 Department of Applied Physics, University of Tokyo, Tokyo 113-8656, Japan \\ 3 Cross Correlated Materials Research Group (CMRG), \\ ASI, RIKEN, WAKO 351-0198, Japan
}

(Dated: October 28, 2018)

\begin{abstract}
We study theoretically the two interacting one-dimensional helical modes at the edges of the quantum spin Hall systems. A new type of inter-edge correlated liquid (IECL) without the spin gap is found. This liquid shows the diverging density wave (DW) and superconductivity (SC) correlations much stronger than those of the spinfull electrons. Possible experimental observations are also discussed.
\end{abstract}


Quantum spin Hall system (QSHS) is a new state of matter topologically distinct from the usual band insulator [1, 2, 3, 4, 5]. It is protected by the band gap induced by the relativistic spin-orbit interaction, and a $Z_{2}$ topological number characterizes it [1, 6]. The simplest picture for QSHS is the two copies of quantum Hall system of up and down pseudospins with the opposite chiralities, and hence the edge modes with the opposite direction of the propagation for different pseudospins along the boundary of the sample are expected [7]. Time-reversal symmetry and Kramer's theorem guarantee the stability of these helical edge modes, i.e., prohibiting the backward scattering between the time-reversal pair states and protecting the crossing of the energy dispersions [8, 9]. These modes, called the helical edge modes, are experimentally observed through the quantized charge conductance $2 e^{2} / h$ in the quantum well of $\mathrm{HgTe} /(\mathrm{Cd}, \mathrm{Hg}) \mathrm{Te}$ [4]. These helical edge modes on one edge are the "half" of the spinfull one-dimensional electrons, because only one chirality is allowed for each pseudospin. Therefore, several nontrivial features are expected for this one-dimensional system such as the half-e charge near the ferromagnetic domain wall [10] and the robustness against the umklapp scattering [8, 9]. Several other theoretical aspects have been studied very recently [11, 12, 13].

In this paper, we study theoretically a new state created by the fusion/recombination of two of the helical edge modes, i.e., the two half spinfull electrons, which is different from the original one-dimensional electrons. This fusion can be realized by the interaction between the edges of the opposite sides of the sample, or one can even design the interacting helical edge modes with the opposite helicity (Fig. 1(a)) or same helicity (Fig. 1(b)). For a moment, we consider the anti-parallel case (Fig. 1(a)), and the translation to the parallel case (Fig. 1(b)) is straightforward and will be discussed later. We employ the bosonization method. There are four fields involved in this problem, i.e., right moving uppseudospin electron density $\rho_{1 R \uparrow}$, and left moving down-pseudospin electron density $\rho_{1 L \downarrow}$, for the edge 1 , and $\rho_{2 R \downarrow}, \rho_{2 L \uparrow}$, for the edge 2 . These densities are related to the phase fields of electrons ( which are defined below Eq.(4) ) $\phi_{1 \uparrow}, \bar{\phi}_{1 \downarrow}, \phi_{2 \downarrow}, \bar{\phi}_{2 \uparrow}$, respectively, as $\rho_{1 R \uparrow}=(1 / \sqrt{\pi}) \partial_{x} \phi_{1 \uparrow}$ etc. [14, 15, 16] (see Fig. 1). This means the spatial variation of the phase corresponds to the accumulation/depletion of the electrons. The novel states induced by the interaction is realized when some combination of these phases becomes rigid, leading to the collective motion of the electrons. We have found that the different combination of $\phi$ 's gets rigid in the two interacting helical edge modes from those realized in the usual 


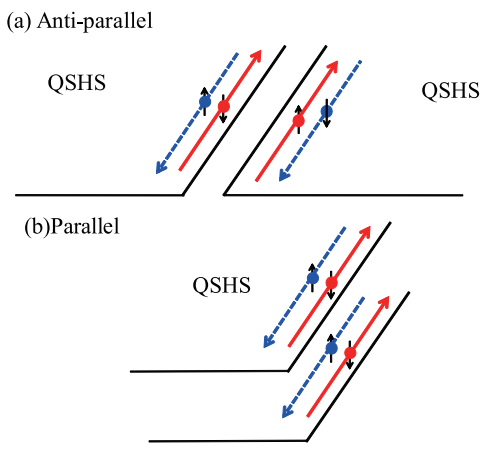

FIG. 1: Schematic illustrations of two helical edge modes with (a)anti-parallel case and (b) parallel case.

spinfull electrons. Namely, the helical edge modes offer an unique opportunity to realize a new state by the recombination of the "fraction" of electrons. To reach this conclusion, we have classified all the possible forms of the interaction, and found that the inter-edge pseudospin flip backward scattering plays an important role in the incommensurate case, while also the inter-edge umklapp scattering can be relevant in the commensurate case. It is found that an inter-edge correlated liquid (IECL) analogous to bipolaron liquid is realized in the incommensurate case, showing the density wave and superconducting correlations both of which are more strongly diverging compared with the one-dimensional spinfull interacting electrons. Furthermore, there is no spin gap in this liquid state, in sharp contrast to the Luther-Emery liquid or the spin gap state in ladder system. Physical properties of this novel liquid are described proposing possible experiments.

The electron-electron interaction is originally given by

$$
H_{\mathrm{int}}=\frac{1}{2} \int d r \int d r^{\prime} \psi_{\sigma}^{\dagger}(r) \psi_{\sigma^{\prime}}^{\dagger}\left(r^{\prime}\right) V\left(r-r^{\prime}\right) \psi_{\sigma^{\prime}}\left(r^{\prime}\right) \psi_{\sigma}(r)
$$

where $r, r^{\prime}$ are the three-dimensional coordinates, $V\left(r-r^{\prime}\right)$ is the interaction potential, and the field operators $\psi_{\sigma}^{\dagger}(r), \psi_{\sigma}(r)$ describe the creation and annihilation of the electron with spin $\sigma$ at the position $r$, respectively. Now we restrict to the helical edge modes only, which means

$$
\psi_{\sigma}(r) \cong \sum_{\alpha=\uparrow, \downarrow} \sum_{a=1,2} c_{\alpha \sigma} \Psi_{a \alpha}(x) \varphi_{a}(y, z) .
$$

Here, the index $a$ specifies the edge, $\sigma$ is the spin of the electron, while $\alpha$ is the pseudospin in the presence of the spin-orbit coupling, and $c_{\alpha \sigma}$ is the coefficient of the transformation. 
$\Psi_{a \alpha}(x)$ represents the field operator of the one-dimensional electrons along the edge channel, while $\varphi_{a}(y, z)$ is the wave function of the helical edge modes perpendicular to the edge $a$. We put this expression into Eq.(11), and neglect the overlap of the wave functions for the helical edge modes at different edges, which means that the different edges can not appear for the same spatial point $r$ or $r^{\prime}$. Also the pseudspin $\alpha$ determines the direction of the propagation for each edge.

When we consider the case where the direct overlap of the wave functions between the two edges can be neglected, the form of the interaction is restricted. The Hamiltonian $H=H_{K}+H_{\mathrm{int}}$ is the sum of the kinetic energy $H_{K}$ and the electron-electron interaction $H_{\text {int }}$ as given by

$$
\begin{aligned}
H_{K} & =-i \int d x\left[\Psi_{1 \uparrow}^{\dagger} \partial_{x} \Psi_{1 \uparrow}-\bar{\Psi}_{1 \downarrow}^{\dagger} \partial_{x} \bar{\Psi}_{1 \downarrow}\right. \\
& \left.+\Psi_{2 \downarrow}^{\dagger} \partial_{x} \Psi_{2 \downarrow}-\bar{\Psi}_{2 \uparrow}^{\dagger} \partial_{x} \bar{\Psi}_{2 \uparrow}\right]
\end{aligned}
$$

and

$$
\begin{aligned}
H_{\mathrm{int}} & =g_{f} \int d x\left[\Psi_{1 \uparrow}^{\dagger} \Psi_{1 \uparrow} \bar{\Psi}_{1 \downarrow}^{\dagger} \bar{\Psi}_{1 \downarrow}+\bar{\Psi}_{2 \uparrow}^{\dagger} \bar{\Psi}_{2 \uparrow} \Psi_{2 \downarrow}^{\dagger} \Psi_{2 \downarrow}\right] \\
& +g_{f}^{\prime} \int d x\left[\Psi_{1 \uparrow}^{\dagger} \Psi_{1 \uparrow} \bar{\Psi}_{2 \uparrow}^{\dagger} \bar{\Psi}_{2 \uparrow}+\bar{\Psi}_{1 \downarrow}^{\dagger} \bar{\Psi}_{1 \downarrow} \Psi_{2 \downarrow}^{\dagger} \Psi_{2 \downarrow}\right] \\
& +g_{u} \int d x\left\{\left[\Psi_{1 \uparrow}^{\dagger}(x) \Psi_{1 \uparrow}^{\dagger}(x+a) \bar{\Psi}_{1 \downarrow}(x+a) \bar{\Psi}_{1 \downarrow}(x)\right.\right. \\
& \left.\left.+\Psi_{2 \downarrow}^{\dagger}(x) \Psi_{2 \downarrow}^{\dagger}(x+a) \bar{\Psi}_{2 \uparrow}(x+a) \bar{\Psi}_{2 \uparrow}(x)\right] \delta+h . c .\right\} \\
& +g_{u}^{\prime} \int d x\left[\Psi_{2 \downarrow}^{\dagger} \Psi_{1 \uparrow}^{\dagger} \bar{\Psi}_{1 \downarrow} \bar{\Psi}_{2 \uparrow} \delta+h . c .\right] \\
& +g_{s f} \int d x\left[\bar{\Psi}_{2 \uparrow}^{\dagger} \Psi_{1 \uparrow}^{\dagger} \bar{\Psi}_{1 \downarrow} \Psi_{2 \downarrow}+\Psi_{2 \downarrow}^{\dagger} \bar{\Psi}_{1 \downarrow}^{\dagger} \Psi_{1 \uparrow} \bar{\Psi}_{2 \uparrow}\right]
\end{aligned}
$$

by right movers (left-movers) $\Psi_{1 \uparrow}$ and $\Psi_{2 \downarrow}\left(\bar{\Psi}_{1 \downarrow}\right.$ and $\left.\bar{\Psi}_{2 \uparrow}\right)$ with $\delta=\exp \left(-i 4 k_{F} x\right) . g_{f}, g_{f}^{\prime}$, $g_{u}, g_{u}^{\prime}$, and $g_{s f}$ denote the interaction constant of intra-edge forward, inter-edge forward, intra-edge umklapp, inter-edge umklapp, and inter-edge pseudospin-flip backward scattering, respectively. The corresponding Hamiltonian with parallel case can be derived by substituting $\bar{\Psi}_{2 \downarrow}$ and $\Psi_{2 \uparrow}$ for $\bar{\Psi}_{2 \uparrow}$ and $\Psi_{2 \downarrow}$.

Fermion operators are expressed by $\Psi_{1 \uparrow}=\eta_{1 \uparrow} \exp \left(-i \sqrt{4 \pi} \phi_{1 \uparrow}\right) / \sqrt{2 \pi}, \quad \bar{\Psi}_{1 \downarrow}=$ $\bar{\eta}_{1 \downarrow} \exp \left(i \sqrt{4 \pi} \bar{\phi}_{1 \downarrow}\right) / \sqrt{2 \pi}, \bar{\Psi}_{2 \uparrow}=\bar{\eta}_{2 \uparrow} \exp \left(i \sqrt{4 \pi} \bar{\phi}_{2 \uparrow}\right) / \sqrt{2 \pi}$, and $\Psi_{2 \downarrow}=\eta_{2 \downarrow} \exp \left(-i \sqrt{4 \pi} \phi_{2 \downarrow}\right) / \sqrt{2 \pi}$, with boson fields $\phi_{1 \uparrow}, \bar{\phi}_{1 \downarrow} \bar{\phi}_{2 \uparrow}$ and $\phi_{2 \downarrow}[15] . \eta_{1 \uparrow}, \bar{\eta}_{1 \downarrow}, \bar{\eta}_{2 \uparrow}$ and $\eta_{2 \downarrow}$ are Klein factors.

Now we consider the bosonization of the Hamiltonians Eq.(3) and Eq.(41) [14]. By introducing $\varphi_{1}=\phi_{1 \uparrow}+\bar{\phi}_{1 \downarrow}, \varphi_{2}=\phi_{2 \downarrow}+\bar{\phi}_{2 \uparrow}, \theta_{1}=\phi_{1 \uparrow}-\bar{\phi}_{1 \downarrow}$, and $\theta_{2}=\phi_{2 \downarrow}-\bar{\phi}_{2 \uparrow}$, the Hamiltonian 
$H$ can be given by

$$
\begin{aligned}
& \frac{v}{2} \int d x\left[\left(\frac{\partial \varphi_{1}}{\partial x}\right)^{2}+\left(\frac{\partial \varphi_{2}}{\partial x}\right)^{2}+\left(\frac{\partial \theta_{1}}{\partial x}\right)^{2}+\left(\frac{\partial \theta_{2}}{\partial x}\right)^{2}\right] \\
& +\frac{g_{f}}{4 \pi} \int d x\left[\left(\frac{\partial \varphi_{1}}{\partial x}\right)^{2}+\left(\frac{\partial \varphi_{2}}{\partial x}\right)^{2}-\left(\frac{\partial \theta_{1}}{\partial x}\right)^{2}-\left(\frac{\partial \theta_{2}}{\partial x}\right)^{2}\right] \\
& +\frac{g_{f}^{\prime}}{2 \pi} \int d x\left[\frac{\partial \varphi_{1}}{\partial x} \frac{\partial \varphi_{2}}{\partial x}-\frac{\partial \theta_{1}}{\partial x} \frac{\partial \theta_{2}}{\partial x}\right] \\
& +\frac{g_{u}}{2 \pi^{2}} \int d x\left\{\cos \left[4\left(\sqrt{\pi} \varphi_{1}-k_{F} x\right)\right]+\cos \left[4\left(\sqrt{\pi} \varphi_{2}-k_{F} x\right)\right]\right\} \\
& +\frac{g_{u}^{\prime}}{2 \pi^{2}} \zeta \int d x \cos \left[\sqrt{4 \pi}\left(\varphi_{1}+\varphi_{2}\right)-4 k_{F} x\right] \\
& -\frac{g_{s f}}{2 \pi^{2}} \zeta \int d x \cos \left[\sqrt{4 \pi}\left(\varphi_{1}-\varphi_{2}\right)\right]
\end{aligned}
$$

with $\zeta=\eta_{2 \downarrow} \eta_{1 \uparrow} \bar{\eta}_{1 \downarrow} \bar{\eta}_{2 \uparrow}$. In order to diagonalize the inter-edge forward scattering terms $\left(g_{f}^{\prime}\right)$, we introduce the symmetric and antisymmetric combinations by $\varphi_{s}^{\prime}=\left(\varphi_{1}+\varphi_{2}\right) / \sqrt{2}, \varphi_{a}^{\prime}=$ $\left(\varphi_{1}-\varphi_{2}\right) / \sqrt{2}, \theta_{s}^{\prime}=\left(\theta_{1}+\theta_{2}\right) / \sqrt{2}$, and $\theta_{a}^{\prime}=\left(\theta_{1}-\theta_{2}\right) / \sqrt{2}$. Then, the above Hamiltonian Eq.(5) can be transformed into $H=H_{0}+H_{u}+H_{u^{\prime}}+H_{s f}$ with each of the term being given by

$$
\begin{aligned}
H_{0} & =\int d x\left\{\frac{v_{1}}{2}\left[\left(\frac{\partial \varphi_{s}^{\prime}}{\partial x}\right)^{2}+K_{1}^{2}\left(\frac{\partial \theta_{s}^{\prime}}{\partial x}\right)^{2}\right]\right. \\
& \left.+\frac{v_{2}}{2}\left[\left(\frac{\partial \varphi_{a}^{\prime}}{\partial x}\right)^{2}+K_{2}^{2}\left(\frac{\partial \theta_{a}^{\prime}}{\partial x}\right)^{2}\right]\right\} \\
H_{u} & =\frac{g_{u}}{\pi^{2}} \int d x \cos \left(\sqrt{8 \pi} \varphi_{s}^{\prime}-4 k_{F} x\right) \cos \left(\sqrt{8 \pi} \varphi_{a}^{\prime}\right), \\
H_{u^{\prime}} & =\frac{g_{u}^{\prime}}{2 \pi^{2}} \zeta \int d x \cos \left[\sqrt{8 \pi} \varphi_{s}^{\prime}-4 k_{F} x\right], \\
H_{s f} & =-\frac{g_{s f}}{2 \pi^{2}} \zeta \int d x \cos \left(\sqrt{8 \pi} \varphi_{a}^{\prime}\right) .
\end{aligned}
$$

The dimensionless parameters $K_{1}$ and $K_{2}$ in Eq. (的) are given by $K_{1}=\sqrt{\frac{2 \pi v-\left(g_{f}+g_{f}^{\prime}\right)}{2 \pi v+\left(g_{f}+g_{f}^{\prime}\right)}}$ and $K_{2}=\sqrt{\frac{2 \pi v-\left(g_{f}-g_{f}^{\prime}\right)}{2 \pi v+\left(g_{f}-g_{f}^{\prime}\right)}}$ with $v_{1}=v+\left(g_{f}+g_{f}^{\prime}\right) /(2 \pi)$ and $v_{2}=v+\left(g_{f}-g_{f}^{\prime}\right) /(2 \pi)$. Note that the difference between $K_{1}$ and $K_{2}$ is due to the presence of inter-edge forward scattering $g_{f}^{\prime}$. Now, we consider the relevance/irrelevance of the nonlinear terms $H_{u}, H_{u^{\prime}}$ and $H_{s f}$. These terms are relevant for $K_{1}+K_{2}<1, K_{1}<1$ and $K_{2}<1$, respectively. For the single edge case, $K_{1}=K_{2}=K, H_{u}$ becomes relevant for $K<1 / 2$ [8], which requires strong repulsive force. The inter-edge interactions $H_{u^{\prime}}$ and $H_{s f}$ can be relevant more easily. The terms $H_{u}$ and $H_{u^{\prime}}$ survive only in the commensurate case; $4 k_{F}=G$ with a reciprocal lattice vector $G$. $H_{s f}$, on the other hand, exists even for the incommensurate case. 
Let us first consider the incommensurate case, which is the most general case because the crossing energy of the helical edge modes are not necessarily at the center of the gap without the particle-hole symmetry, and also the Fermi energy is often determined by the details of the sample, e.g., in-gap impurity levels and does not give the commensurate filling of the edge channels. In this case, only the inter-edge pseudospin-flip backward scattering $g_{s f}$ is relevant for $K_{2}<1$, which is realized when the intra-edge repulsive forward scattering $g_{f}(>0)$ is stronger than the inter-edge repulsion $g_{f}^{\prime}(>0)$ for example. This relevant $g_{s f}$ term fixes $\varphi_{a}^{\prime}$ to open the gap for its fluctuation. The remnant massless modes are the conjugate pair $\varphi_{s}^{\prime}$ and $\theta_{s}^{\prime}$, which correspond to the correlated motion of the charge/spin between the two edges analogous to the bipolaron formation in spinfull ladder system [16, 17].

We first focus on the generic phase diagram. It is determined by comparing the critical exponents of diverging correlation function of the following four order parameters, i.e., (i)Intra-edge Density wave (DW), $\Psi_{1 \uparrow}^{\dagger} \bar{\Psi}_{1 \downarrow} \pm \Psi_{2 \downarrow}^{\dagger} \bar{\Psi}_{2 \uparrow}$; (ii)Inter-edge DW, $\Psi_{1 \uparrow}^{\dagger} \bar{\Psi}_{2 \uparrow} \pm \bar{\Psi}_{1 \downarrow}^{\dagger} \Psi_{2 \downarrow}$; (iii) Intra-edge Superconductivity (SC) $\Psi_{1 \uparrow} \bar{\Psi}_{1 \downarrow} \pm \Psi_{2 \downarrow} \bar{\Psi}_{2 \uparrow}$, and (iv)Inter-edge SC, $\Psi_{1 \uparrow} \bar{\Psi}_{2 \uparrow} \pm \bar{\Psi}_{1 \downarrow} \Psi_{2 \downarrow}$. Using the standard bosonization method, the correlation function of each of the above order parameters behaves as

$$
<O(x, \tau) O(0,0)>\sim\left[\max \left(|x|, v_{i=1,2}|\tau|\right)\right]^{-\nu}
$$

in the presence of gapless modes. Figure 2 shows the phase diagram in the plane of $g_{f}$ and $g_{f}^{\prime}$. The phase boundaries, which are the straight lines $g_{f}=g_{f}^{\prime}, g_{f}=-g_{f}^{\prime}$ correspond to $K_{2}=1, K_{1}=1$, respectively. Namely, each region is characterized by $(\mathrm{A}) K_{1}<1, K_{2}<1$, (B) $K_{1}>1, K_{2}<1$, (C) $K_{1}>1, K_{2}>1$, and (D) $K_{1}<1, K_{2}>1$, respectively. Table I and Table II explain the behaviors of the order parameter correlation functions for the incommensurate and commensurate cases, respectively. For incommensurate case (Table I), $H_{s f}$ is relevant and IECL is realized in regions A and B. In this case, only the (i) Intraedge DW or (iv) Inter-edge SC shows the critical power-law behavior, while the others are exponentially decaying (" - " symbol in Table I). The most dominant order in each region is indicated by the bold letters, i.e., (i)Intra-edge DW in region A, (ii)Inter-edge DW in region $\mathrm{D}$, (iii)Intra-edge $\mathrm{SC}$ in region $\mathrm{C}$, and (iv)Inter-edge $\mathrm{SC}$ in region $\mathrm{B}$.

We further study the physical properties of the IECL other than the order parameters. First, we consider the stability of this state against the inter-edge tunneling which has been 


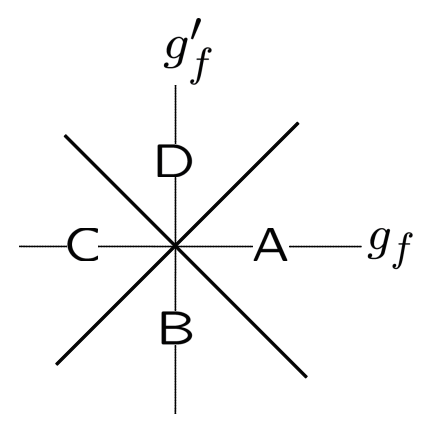

FIG. 2: Phase diagram of two helical edge modes. Intra-edge Density wave (DW), Inter-edge Superconductivity (SC), Intra-edge SC, and Inter-edge DW, are most dominant in the region A, $\mathrm{B}, \mathrm{C}$, and D, respectively.

\begin{tabular}{|c|c|c|c|c|}
\hline Region & A & B & C & $\mathrm{D}$ \\
\hline state & \multicolumn{2}{|c|}{ IECL } & \multicolumn{2}{|c|}{$\mathrm{TL}$} \\
\hline $\begin{array}{l}\text { Intra } \\
\text { DW }\end{array}$ & $K_{1}$ & $K_{1}$ & $K_{1}+K_{2}$ & $K_{1}+K_{2}$ \\
\hline $\begin{array}{l}\text { Inter } \\
\text { DW }\end{array}$ & \multicolumn{2}{|c|}{-} & $K_{1}+1 / K_{2}$ & $K_{1}+1 / K_{2}$ \\
\hline $\begin{array}{l}\text { Intra } \\
\mathrm{SC}\end{array}$ & \multicolumn{2}{|c|}{-} & $1 / K_{1}+1 / K_{2}$ & $1 / K_{1}+1 / K_{2}$ \\
\hline Inter SC & $1 / K_{1}$ & $1 / K_{1}$ & $1 / K_{1}+K_{2}$ & $1 / K_{1}+K_{2}$ \\
\hline
\end{tabular}

TABLE I: The relation between the most dominant order parameters and the exponents of correlation function for incommensurate case. " - "denotes that the correlation function has an exponential dependence. The most dominant order in each region is indicated by the bold letters.

neglected so far. The Hamiltonian describing this process is given by

$$
H_{t}=-\frac{2}{\pi} \int d x\left\{t \cos \left[\sqrt{2 \pi}\left(\varphi_{s}^{\prime}+\theta_{a}^{\prime}\right)\right]-t^{\prime} \cos \left[\sqrt{2 \pi}\left(\varphi_{a}^{\prime}+\theta_{a}^{\prime}\right)\right]\right\}
$$

where $t$ and $t^{\prime}$ are transfer integrals without and with pseudospin-flip scattering, respectively. It is evident that this perturbation is irrelevant since $\varphi_{a}^{\prime}$ is fixed in IECL.

Next, we consider the magnetic properties of IECL. In this state, the spin-spin correlation function has two contributions, $<S(x=0, \tau) S(x=0,0)>\sim \frac{1}{\tau^{2}}+\left(\frac{1}{\tau}\right)^{K_{1}}$. The Fourier transform with respect to the imaginary time $\tau$ thus leads to the spin susceptibility $\chi_{s} \sim$ 
$T+T^{K_{1}-1}$ as a function of temperature $T$ [16]. Then the resulting temperature dependence of NMR relaxation rate $T_{1}$ is given by $\frac{1}{T_{1}} \sim T+T^{K_{1}-1}$. It should be remarked that $1 / T_{1}$ has a divergent $T$ dependence as $T \rightarrow 0$ for $K_{1}<1$, where Intra-edge DW state is dominant realized in region A. This anomalous temperature dependence of $T_{1}$ [18] can be detected by experiments of NMR.

The Josephson coupling through IECL shows also a novel property. Here, we consider the IECL with length $d$ is sandwiched by two superconductors $\mathrm{S}_{1}$ and $\mathrm{S}_{2}[19]$. The Josephson current $I_{J}$ is given by $I_{J}=-2 e \partial F(\chi) / \partial \chi$ with phase difference $\chi$. The phase dependent part of the free energy is proportional to $F(\chi) \propto \operatorname{Re}\left[t_{1}^{2}\left(t_{2}^{*}\right)^{2} e^{-i \chi} \int_{0}^{\beta} d \tau \Pi(d, \tau)\right]$ with $\Pi(d, \tau)=<O(0,0) O(d, \tau)>, O(0,0)=\Psi_{1 \uparrow} \bar{\Psi}_{2 \uparrow} \pm \bar{\Psi}_{1 \downarrow} \Psi_{2 \downarrow}$ and $\beta=1 / T . t_{1}\left(t_{2}\right)$ is the transmission amplitude at IECL $/ \mathrm{S}_{1}\left(\mathrm{~S}_{2}\right)$ interface. Following the discussion by Fazio et al. [19], the resulting $I_{J}$ at zero temperature is proportional to $d^{-1 / K_{1}+1}$ for $K_{1}<1$. This is quite unusual that Josephson current is hugely enhanced as compared to conventional spinfull Luttinger chain where $I_{J}$ is proportional to $d^{-1 / K}$.

\begin{tabular}{|l|l|l|l|l|}
\hline Region & $\mathrm{A}$ & $\mathrm{B}$ & $\mathrm{C}$ & $\mathrm{D}$ \\
\hline state & $\mathrm{DW}$ & $\mathrm{IECL}$ & $\mathrm{TL}$ & IECL \\
\hline Intra & Order & $K_{1}$ & $K_{1}+K_{2}$ & $K_{2}$ \\
DW & & & & \\
\hline Inter & - & - & $K_{1}+1 / K_{2}$ & $\mathbf{1} / \boldsymbol{K}_{\mathbf{2}}$ \\
DW & & & & \\
\hline Intra SC & - & - & $\mathbf{1} / \boldsymbol{K}_{\mathbf{1}}+\mathbf{1} / \boldsymbol{K}_{\mathbf{2}}$ & - \\
\hline Inter SC & - & $\mathbf{1} / \boldsymbol{K}_{\mathbf{1}}$ & $1 / K_{1}+K_{2}$ & - \\
\hline
\end{tabular}

TABLE II: Similar Table to Table I for the commensurate case.

Now we turn to the commensurate case (Table II). The term $H_{u^{\prime}}$ [Eq. (8)] fixes the phase $\varphi_{s}^{\prime}$ for $K_{1}>1$ which is realized in regions A and D. The Intra DW phase is stabilized in the region $\mathrm{A}$ and order parameter has a nonzero value, i.e., long-range ordered state at zero temperature. The Inter DW is also stabilized and the resulting $\nu$ becomes $1 / K_{2}$ in the region $\mathrm{D}$. On the other hand, the exponents $\nu$ 's in the regions $\mathrm{B}$ and $\mathrm{C}$ do not change from 
the incommensurate case, since the umklapp terms is irrelevant there. Note that the most dominant order parameter in each region does not change from the incommensurate case.

In the parallel helical edge modes (Fig.1(b)), we can obtain essentially the same results as the anti-parallel case discussed thus far. The only difference is that the corresponding operators for (ii)Inter-edge charge density wave (CDW)/ spin density wave (SDW) and (iv)Inter-edge SC are given as $\Psi_{1 \uparrow}^{\dagger} \bar{\Psi}_{2 \downarrow} \pm \bar{\Psi}_{1 \downarrow}^{\dagger} \Psi_{2 \uparrow}$ and $\Psi_{1 \uparrow} \bar{\Psi}_{2 \downarrow} \pm \bar{\Psi}_{1 \downarrow} \Psi_{2 \uparrow}$. This means that the opposite pseudospin electron operators appear in the parallel case instead of the same pseudospin ones in the anti-parallel case.

Finally, we compare the present IECL with the one-dimensional liquids already known. The single edge mode case is reproduced by setting $g_{f}^{\prime}=0, g_{u}^{\prime}=0$ and $g_{s f}=0$. Then $K_{1}=K_{2}=K$ is satisfied. The intra-edge umklapp scattering $g_{u}$ becomes relevant for $K<1 / 2[8]$. The exponent of correlation function $\nu$ of Intra-edge SC and Intra-edge DW are given by $2 / K$ and $2 K$, which shows weaker correlation compared with those in Table I and II. For spinfull one-dimensional TL liquid, the $\nu$ of Intra-edge SC and Intra-edge $\mathrm{CDW} / \mathrm{SDW}$ are given by $1 / K_{\rho}+K_{\sigma}\left(1 / K_{\sigma}\right)$ and $K_{\rho}+K_{\sigma}\left(1 / K_{\sigma}\right)[16]$. As compared to this spinfull one-dimensional TL liquid, the density wave (SDW/CDW) and superconducting correlations both of which are more strongly diverging. The spin gap phase is realized in Luther-Emery liquid [16, 20]. It is known that the $\nu$ of the intra-edge SC and the intra-edge CDW are given by $1 / K$ and $K$, respectively. Although these exponents are similar to those in IECL, IECL is very much different since there is no spin gap. This difference comes from the fact that the combination $\phi_{1 \uparrow}+\bar{\phi}_{2 \uparrow}-\phi_{2 \downarrow}-\bar{\phi}_{1 \downarrow}$ is fixed in Luther-Emery liquid while a different one $\phi_{1 \uparrow}-\bar{\phi}_{2 \uparrow}-\phi_{2 \downarrow}+\bar{\phi}_{1 \downarrow}$ is fixed in IECL. The present IECL is also different from spinfull two-leg ladder, where the $\nu$ of the inter-ladder $d$-wave pairing can become $1 /(2 K)$ [21]. However, there opens the spin gap also in this state, in sharp contrast to the present IECL.

In this letter, we have proposed an Inter-edge correlated liquid (IECL) for the two helical edge modes of quantum spin Hall systems. IECL is realized most probably in the incommensurate case, showing the density wave and superconducting correlations both of which are more strongly diverging compared with the one-dimensional spinfull interacting electrons. Furthermore, there is no spin gap in this liquid state, in sharp contrast to the Luther-Emery liquid or the spin gap state in two-leg ladder system. This state can be clearly identified experimentally by NMR and Josephson junctions. 
This work is partly supported by the Grant-in-Aids from under the Grant No. 20654030, No. 19048015 and No. 19048008 from MEXT, Japan, and NTT basic research laboratories.

[1] C. L. Kane and E. J. Mele, Phys. Rev. Lett. 95, 146802 (2005); C. L. Kane and E. J. Mele, Phys. Rev. Lett. 95, 226801 (2005).

[2] B. A. Bernevig, and S. C. Zhang, Phys. Rev. Lett. 96, 106802 (2006).

[3] B. A. Bernevig, T. L. Hughes, and S. C. Zhang, Science 3141757 (2006).

[4] M. König, et. al. Science, 318, 766 (2007); M. König, et. al. J. Phys. Soc. Jpn. 77031007 (2008).

[5] M. Onoda and N. Nagaosa, Phys. Rev. Lett. 95, 106601 (2005).

[6] L. Fu and C. L. Kane, Phys. Rev. B 74, 195312 (2006); L. Fu and C. L. Kane, Phys. Rev. B 76, 045302 (2007).

[7] X.G. Wen, Phys. Rev. B 445708 (1991).

[8] C. Wu, B.A. Bernevig, and S.C. Zhang, Phys. Rev. Lett. 96 106401, (2006).

[9] C. Xu and J. E. Moore, Phys. Rev. B 73 045322, (2006).

[10] X.-L. Qi, T. Hughes, and S.-C. Zhang, Nature Physics, 4273 (2008).

[11] J. Maciejko, C. Liu, Y. Oreg, X-L. Qi, C. Wu and S.C. Zhang, arXiv: 0901.1685.

[12] C.Y. Hou, E.A. Kim, and C. Chamon, Phys. Rev. Lett. 102076602 (2009).

[13] A. Ström and H. Johannesson, Phys. Rev. Lett. 102096806 (2009).

[14] J. Solyom, Advances in Physcis, 28209 (1979).

[15] D. Senechal, arXiv:condmat[9908262].

[16] T. Giamarchi. Quantum Physics in one-dimension (Oxford Science Publications, 2004).

[17] N. Nagaosa, Solid State Commun. 94495 (1995).

[18] G. Chaboussant, et. al., Phys. Rev. Lett. 80, 2713 (1998).

[19] R. Fazio, F.W.J. Hekking and A.A. Odintsov, Phys. Rev. Lett. 74, 1843 (1995).

[20] In the paper V. Gritsev, G. Japaridze, M. Pletyukhov, and D. Baeriswyl, Phys. Rev. Lett. 94137207 (2005), the one-dimensional spinfull TL liquid with Rashba interaction has been discussed. They considered the similar term to $H_{s f}$ in Eq.(9), and obtained the spin gap state. However, the chirality is determined by the spin for each edge, which is not the case in their model. Therefore, their spin-gap state is quite different from the IECL described here. 
[21] L. Balents and M.P.A Fisher, Phys. Rev. B 5312133 (1996); E. Orignac and T. Giamarchi, Phys. Rev. B 567167 (1997). 